\section{Influenza: Neue STIKO-Empfehlung}

Die Ständige Impfkommission (STIKO) hat ihre bisherige Empfehlung zum Schutz gegen saisonale Influenza präzisiert und spricht sich nun für einen quadrivalenten Impfstoff aus. Das hat das Gremium am 14. November 2017 beschlossen. Die Empfehlung tritt im kommenden Februar mit der Publikation im "Epidemiologischen Bulletin“ in Kraft. Der Gemeinsame Bundesausschuss wird dann binnen drei Monaten entscheiden, ob die Impfung GKV-Pflichtleistung wird. Die Empfehlung gilt für alle Personen, für die die saisonale Influenzaimpfung von der STIKO empfohlen wird.

wWw.rki.de

\section{„Iss doch Schokolade"}

In Deutschland gibt es mit Blick auf das Krankheitsbild Depression große Wissenslücken. So findet nach einer repräsentativen Umfrage fast jeder Fünfte, dass sich Betroffene zusammenreißen sollten, heißt es im „Deutschland-Barometer Depression" der Stiftung Deutsche Depressionshilfe und der Deutsche Bahn Stiftung. Ein weiteres knappes Fünftel hält Schokolade für ein geeignetes Hilfsmittel gegen Depressionen. Nahezu alle Interviewten sahen die Ursache einer Depression in Schicksalsschlägen (96\%) und Belastungen am Arbeitsplatz (94\%). Die Hälfte glaubte an eine falsche Lebensführung, ein Drittel an Charakterschwäche.

www.deutsche-depressionshilfe.de

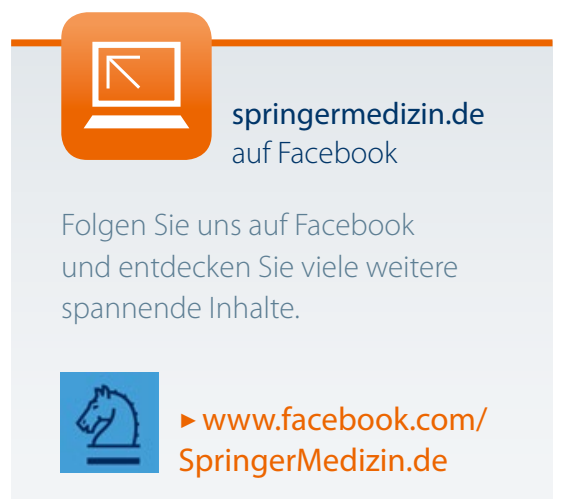

Vergleich mit Schwimmen und Laufen

\title{
Radfahren schadet nicht der Potenz - aber ...
}

Im Vergleich zu Schwimmern und Läufern haben Radfahrer keine schlechtere Sexualfunktion. Auch sonst schadet das Radeln dem Urogenitaltrakt nicht - mit einer wichtigen Ausnahme.

Für eine Querschnittstudie hat eine amerikanische Forschergruppe knapp 4000 Männer auf Erektions- und urogenitale Störungen befragt. Etwa 2800 von ihnen waren Radfahrer, die übrigen Schwimmer oder Läufer. Signifikante Unterschiede bei den Symptomenscores für Beschwerden des unteren Harntraktes zwischen Radfahrern und Nichtradfahrern waren nicht festzustellen. Mit Blick auf die Erektionsfunktion standen die Radfahrer sogar etwas besser da als Schwimmer und Läufer.

Radfahrer hatten aber etwa dreimal so häufig Harnröhrenstrikturen wie Nichtradfahrer. Interessanterweise galt dies jedoch nicht für die Intensivradler. Warum dies so ist, darüber können die amerikanischen Forscher nur spekulieren. Möglicherweise radeln ehemalige Strikturträger nicht mehr so viel und fallen daher nicht mehr in die Gruppe der Vielfahrer. Oder aber die Langstreckenradfahrer wissen perineale Verletzungen aufgrund größerer Erfahrung besser zu vermeiden.

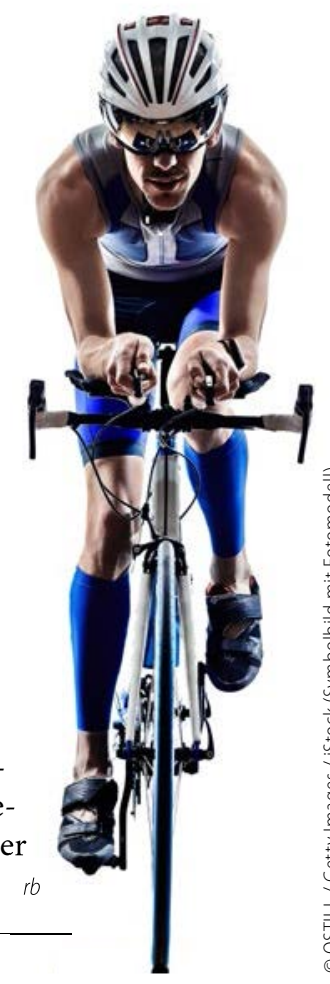

Awad MA et al. J Urol 2017, online 13. Oktober

https://doi.org/10.1016/j.juro.2017.10.017

\section{Mehr als ein Placeboeffekt}

\section{Fremdstuhl kann Reizdarm beruhigen}

Typische Beschwerden eines Reizdarms lassen sich durch die Übertragung von Stuhl eines gesunden Spenders deutlich reduzieren.

Schon länger wird vermutet, dass Patienten mit Reizdarmsyndrom (Irritable Bowel Syndrome, IBS) von einer fäkalen Mikrobiotatransplantation (FMT) profitieren könnten. Die erste randomisierte placebokontrollierte Studie in dieser Indikation bestätigt diese Hoffnung.

Teilnehmer waren 90 Patienten mit einem mindestens mittelschweren IBS (IBSSeverity Scoring System (IBS-SSS) $\geq 175$ Punkte). 60 Patienten wurden einer aktiven, 30 einer Placebo-FMT zugeteilt. Für die aktive Therapie wurde Stuhl von zwei gesunden Spendern verwendet, für die Scheintherapie patienteneigener Stuhl.

Eine Reduktion des IBS-SSS um mehr als 75 Punkte nach drei Monaten erreichten $65 \%$ der Patienten mit aktiver und 43\% mit Placebobehandlung, ein gerade signifikanter Unterschied ( $p=0,049)$. Die Ergebnisse passen zu dem Konzept, wonach die Pathophysiologie beim Reizdarm „eng mit einer Störung der Darmmikrobiota zusammenhängt", wie die Studienautoren schreiben.

Johnsen PH et al. Lancet Gastroenterol Hepatol 2017, online 31. Oktober, https://doi.org/10.1016/S2468-1253(17)30338-2 